\title{
Mapping quantitative trait loci for heat tolerance at the booting stage using chromosomal segment substitution lines in rice
}

\author{
Shan Zhu ${ }^{1,2} \cdot$ Renliang Huang ${ }^{2} \cdot$ Hnin Pwint Wai ${ }^{2,3} \cdot$ Hongliang Xiong ${ }^{2} \cdot$ \\ Xianhua Shen ${ }^{2} \cdot$ Haohua $\mathrm{He}^{1} \cdot$ Song Yan $^{2}$
}

Received: 17 March 2017/Revised: 15 August 2017/Accepted: 25 August 2017/Published online: 21 September 2017

(c) Prof. H.S. Srivastava Foundation for Science and Society 2017

\begin{abstract}
High temperature stress is a major obstacle in rice productivity. Considerable progress has been made on studying heat tolerance (HT) at different stages. However, the genetic basis of HT at the booting stage is poorly understood. In this study, we analyzed the morphological features of a heat-sensitive japonica cultivar Sasanishiki under natural high temperature stress at the booting stage. The anthers became smaller and the number, and fertility, of pollen grains were decreased significantly. As a result, there was a dramatic reduction in spikelet fertility. In contrast, the indica cultivar Habataki showed high HT and normal spikelet fertility under high temperature stress. Additonally, a set of chromosome segment substitution lines, derived from Sasanishiki and Habataki, were
\end{abstract}

Shan Zhu and Renliang Huang have contributed equally to this work.

Electronic supplementary material The online version of this article (doi:10.1007/s12298-017-0465-4) contains supplementary material, which is available to authorized users.

Haohua $\mathrm{He}$

hhhua64@163.com

$\triangle$ Song Yan

yans11@163.com

1 Key Laboratory of Crop Physiology, Ecology and Genetic Breeding, Ministry of Education, College of Agronomy, Jiangxi Agricultural University, Nanchang 330045, China

2 Rice National Engineering Laboratory (Nanchang), Nanchang Sub-center, National Rice Improvement Center, Jiangxi Provincial Key Laboratory of Rice Physiology and Genetics, Rice Research Institute, Jiangxi Academy of Agricultural Sciences, Nanchang 330200, China

3 Biotechnology Research Department, Department of Research and Innovation, Ministry of Education (Ministry of Science and Technology), Kyaukse 05151, Myanmar evaluated for HT related quantitative trait loci (QTLs) across two environments in the natural field. A total of 12 QTLs associated with HT were detected, of which, 5 were identified in two environments, and 7 in one environment. Furthermore, one of the major-effect QTLs (qHTB3-3) detected on the long arm of chromosome 3 , was confirmed using overlapping substituted lines. qHTB3-3 was finally mapped between the two markers RM3525 and 3-M95, approximately $2.8 \mathrm{Mb}$ apart. These findings and further gene cloning of $q H T B 3-3$ will help us better understand the molecular control of HT in rice, and may contribute to the development of high HT rice varieties.

Keywords Rice (Oryza sativa L.) · Heat tolerance · Booting stage $\cdot$ CSSL $\cdot$ QTL $\cdot q H T B 3-3$

\section{Introduction}

Rice is one of the thermophilic crops. Optimal temperature for the normal development of rice is between 27 and $32{ }^{\circ} \mathrm{C}$ (Yin et al. 1996). Temperature above the tolerance threshold of growth can cause heat stress. Unfortunately, high temperature events have increased resulting in reduced rice yields in recent years (Shi et al. 2015). According to the investigation on high-temperature events, occurrence of rice heat stress increased significantly from 1983 to 2010 in Jiangxi province, China (Yang et al. 2012). Two severe heat stress events occurred during the flowering, or grain-filling stage, in 2003 and 2013. These events caused a great reduction of rice yield in many countries of south China (Tao et al. 2007; Gong and Shao 2013). As extreme high temperature events occur more frequently, it is becoming much more important to breed heat-tolerant varieties or identify varieties from pre-existing germplasm. 
Two strategies have been proposed for breeding. One is to use the highly heat-tolerant cultivars as the donor, such as N22 (Rang et al. 2011). Another is to breed cultivars with the early-morning flowering (EMF) trait, to escape high temperature at flowering. $q E M F 3$, which was identified recently from wild rice $O$. officinalis, has the potential to shift flower opening time of cultivars to an earlier time in morning, when the temperature is lower (Hirabayashi et al. 2015)

In rice, the flowering stage is the most susceptible to high temperatures, followed by the booting stage (Satake and Yoshida 1978; Shah et al. 2011). Many studies have focused on HT at the flowering stage. For example, spikelet sterility was induced in two rice cultivars (IR64 and Azucena) exposed to $\geq 33.7^{\circ} \mathrm{C}$, for more than $1 \mathrm{~h}$ at anthesis (Jagadish et al. 2007). High temperature stress causes bad anther dehiscence and represses germination of pollen grains on the stigma (Matsui and Omasa 2002; Prasad et al. 2006; Jagadish et al. 2010). Interestingly, female fertility was more tolerant to high temperature, as long as the environmental temperature was below $41{ }^{\circ} \mathrm{C}$ (Satake and Yoshida 1978). Additionally, QTL mapping studies for HT at flowering stage have been carried out on various rice populations. A number of related QTLs have been identified and located on all 12 chromosomes, showing a complex genetic basis of HT (Chen et al. 2008; Zhang et al. 2008; Xiao et al. 2011; Ye et al. 2012, 2015; Buu et al. 2014; Zhao et al. 2016; Prasanth et al. 2016). One major QTL (TT1) was recently cloned from African rice (O. glaberrima), which encodes an $\alpha 2$ subunit of the $26 \mathrm{~S}$ proteasome involved in the degradation of ubiquitinated proteins. TTl has shown great value in improving HT at seedling, flowering and filling stages (Li et al. 2015).

Much progress has been made on HT at the flowering stage; however, few studies focus on HT at booting stage. High temperature $\left(>33{ }^{\circ} \mathrm{C}\right)$ at the meiosis stage affects spikelet fertility. With the increase in temperature, and its duration, the seed-setting rate gradually decreases (Shi et al. 2008). Zhao et al. (2006) mapped 2 QTLs for the heat susceptibility index of spikelet fertility at the booting stage

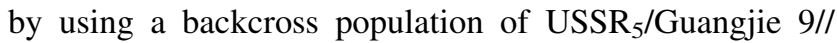
USSR $_{5}$. The two QTLs (located on chromosome 4 and 8) explained 16.8 and $9.9 \%$ of the phenotypic variance, respectively. There has been no systematic analysis performed on HT of rice at the booting stage. Here, we studied the performance of a heat-sensitive japonica cultivar Sasanishiki under high temperature stress at the booting stage. Furthermore, a set of CSSLs, derived from the backcross between Sasanishiki and an indica donor cultivar Habataki, were used to detect QTLs associated with HT at the booting stage.

\section{Materials and methods}

\section{Plant materials}

A set of 39 CSSLs, derived from the backcrossed progenies involving the japonica variety Sasanishiki (recurrent parent) and an indica elite variety Habataki (donor parent) (Ando et al. 2008), were used. In 2016, CSSLs and their parents were grown in the paddy field of Jiangxi Academy of Agricultural Sciences, Nanchang, China. Field experiments consisted of four sowing dates (March 24, 30, May 17 and 23, 2016), hereafter referred to as E1, E2, E3 and $\mathrm{E} 4$, respectively. The seedlings from $\mathrm{E} 1$ and $\mathrm{E} 2$ were transplanted on April 27, and the others were transplanted on June 16. Each line was planted in 5 rows, with 8 plants per row, spaced at $20 \times 20 \mathrm{~cm}^{2}$. The field management was conducted according to the normal procedures for rice.

\section{Phenotypic measurements}

Air temperature in the field was recorded from 1 July to 30 August 2016 using a copper-constantan thermocouple (Zeda Instruments Co. LTD, Hangzhou, China). The details of temperature at the booting stage are shown in Fig. 1. Pollen fertility was observed under an optical microscope with $1 \% \quad \mathrm{I}_{2}-\mathrm{IK}$ staining method at flowering prior to anthesis. Images were captured using a digital microscope camera (Motic China Group Co., Ltd.). The spikelet fertility under different environments was used as indicator of tolerance to high temperature for the QTL mapping. At maturity, 15 panicles per line were randomly harvested for spikelet fertility investigation.

\section{QTL detection}

A QTL was considered to be present, when the average value of a trait was significantly different between a CSSL and the recurrent parent (Sasanishiki) according to Dunnett's multiple comparison test using the SPSS software. The additive effect and percentage of phenotypic variance explained by each QTL independently, were also calculated (Eshed and Zamir 1995). The positive additive effect indicates that Habataki contributes to the positive allele, whereas the negative additive effect indicates that the positive allele is contributed by Sasanishiki.

\section{QTL validation}

A cross was made between SL411 and Sasanishiki. SSR markers and new developed insertion/deletion (indel) makers were used to identify the progenies. Finally, the backcross inbred lines (BILs) were obtained from the $F_{3}$ 


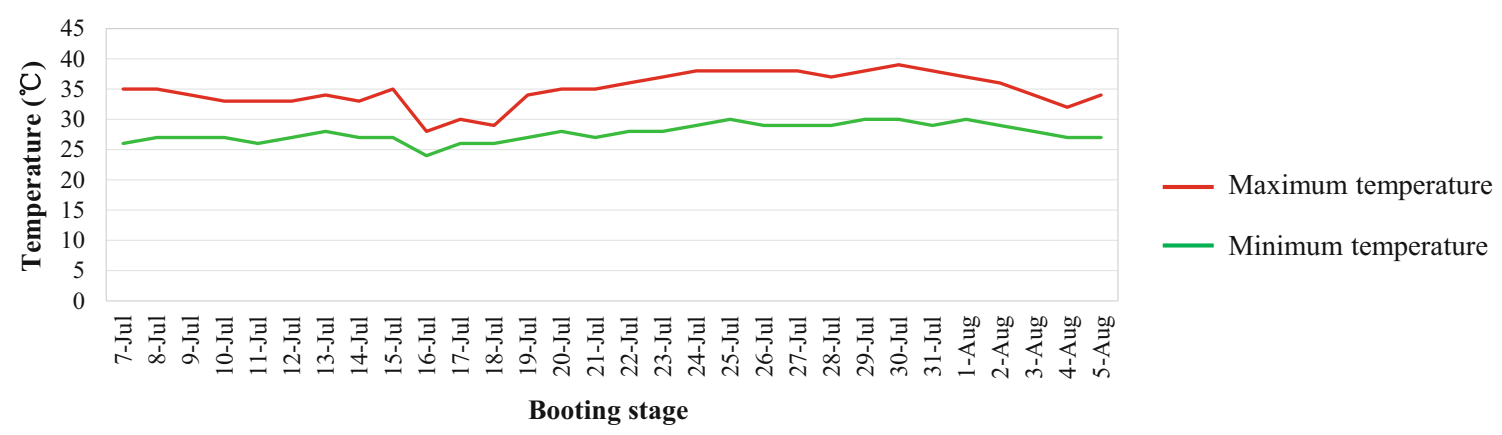

Fig. 1 Maximum and minimum temperatures recorded at the booting stage in 2016

generation. The BILs were treated the same as CSSLs in E3 and E4. Primers were designed using Primer3 (Untergasser et al. 2012) and synthesized by Genscript Technology (Nanjing) Co., Ltd (Table S1). The PCR program included 4 min at $94{ }^{\circ} \mathrm{C}$, followed by 32 cycles at $94{ }^{\circ} \mathrm{C}$ for $20 \mathrm{~s}, 55^{\circ} \mathrm{C}$ for $20 \mathrm{~s}$, and $72{ }^{\circ} \mathrm{C}$ for $30 \mathrm{~s}$, and a final extension at $72{ }^{\circ} \mathrm{C}$ for $5 \mathrm{~min}$. PCR products were separated on $3.5 \%$ agarose gels or $6 \%$ polyacrylamide gels stained with Gel Red or silver, respectively.

\section{Results}

\section{The spikelet fertility of CSSLs in E1 and E2}

In E1 and E2, there was no extreme high temperature during the process of rice growth. The two parents, Sasanishiki and Habataki, showed normal spikelet fertility (Table 1). Of the 39 CSSLs, 37 lines (SL401-SL437) showed normal spikelet fertility. Therefore, E1 and E2 could be served as normal growth conditions. However, SL438 and SL439 showed very low seed-set percentage with 23.84 and $22.19 \%$, respectively. Because the spikelet fertility would be used as a screening index for HT, the two lines obviously contained genetic factors decreasing the spikelet fertility under normal conditions, thus they could not reflect real effects on HT and were not considered for further investigation under high temperature.

\section{Heading dates of CSSLs and high temperature at the booting stage in $\mathrm{E3}$ and $\mathrm{E} 4$}

In E3, Sasanishiki and Habataki headed on 29 July and 2 August 2016, respectively. The heading dates of CSSLs varied between 28 July to 2 August, except SL428, which headed on 5 August. In E4, all lines including the two parents headed about 3 days later than in E3. Small variations in heading time existed between CSSLs in each condition, and all lines showed different reductions of spikelet fertility.

Generally, the booting stage is about 30 days before heading in rice. Therefore, to assess the impact of high temperatures at the booting stage, the period from 7 July to 5 August 2016 was considered as the booting stage. During this period, the highest daily temperature reached $39^{\circ} \mathrm{C}$ and temperatures above $37^{\circ} \mathrm{C}$ lasted for 10 days (Fig. 1), which might affect the floral organ development. To examine whether the CSSLs suffered from high temperatures at flowering stage, the temperatures of 15 days from 1 August to 15 August 2016 were checked. The temperatures appeared lower than that at the booting stage, and moderate daily high-temperatures below $35^{\circ} \mathrm{C}$ lasted for 8 days from 3 August to 10 August (Table S2). In addition, no extreme high temperatures were observed at the filling stage. Thus, it can be concluded that the reductions in spikelet fertility were due to the high temperatures at the booting stage.
Table 1 Phenotype data of spikelet fertility under high temperature for CSSLs (SL401SL437) and parents (Sasanishiki and Habataki) across four environments

\begin{tabular}{|c|c|c|c|c|c|}
\hline \multirow[t]{2}{*}{ Environment } & \multicolumn{2}{|l|}{ Parents } & \multicolumn{3}{|l|}{ CSSLs } \\
\hline & Sasanishiki (\%) & Habataki (\%) & Mean $(\%)$ & $\operatorname{Min}(\%)$ & $\operatorname{Max}(\%)$ \\
\hline E1 & 96.99 & 95.11 & 95.50 & 93.21 & 98.77 \\
\hline E2 & 98.47 & 94.36 & 96.17 & 93.79 & 98.85 \\
\hline E3 & 19.71 & $84.36 * *$ & 30.51 & 12.25 & 58.60 \\
\hline E4 & 18.56 & $82.40 * *$ & 23.23 & 5.99 & 56.62 \\
\hline
\end{tabular}

** Mean the significance levels of 0.01 between Habataki and Sasanishiki 

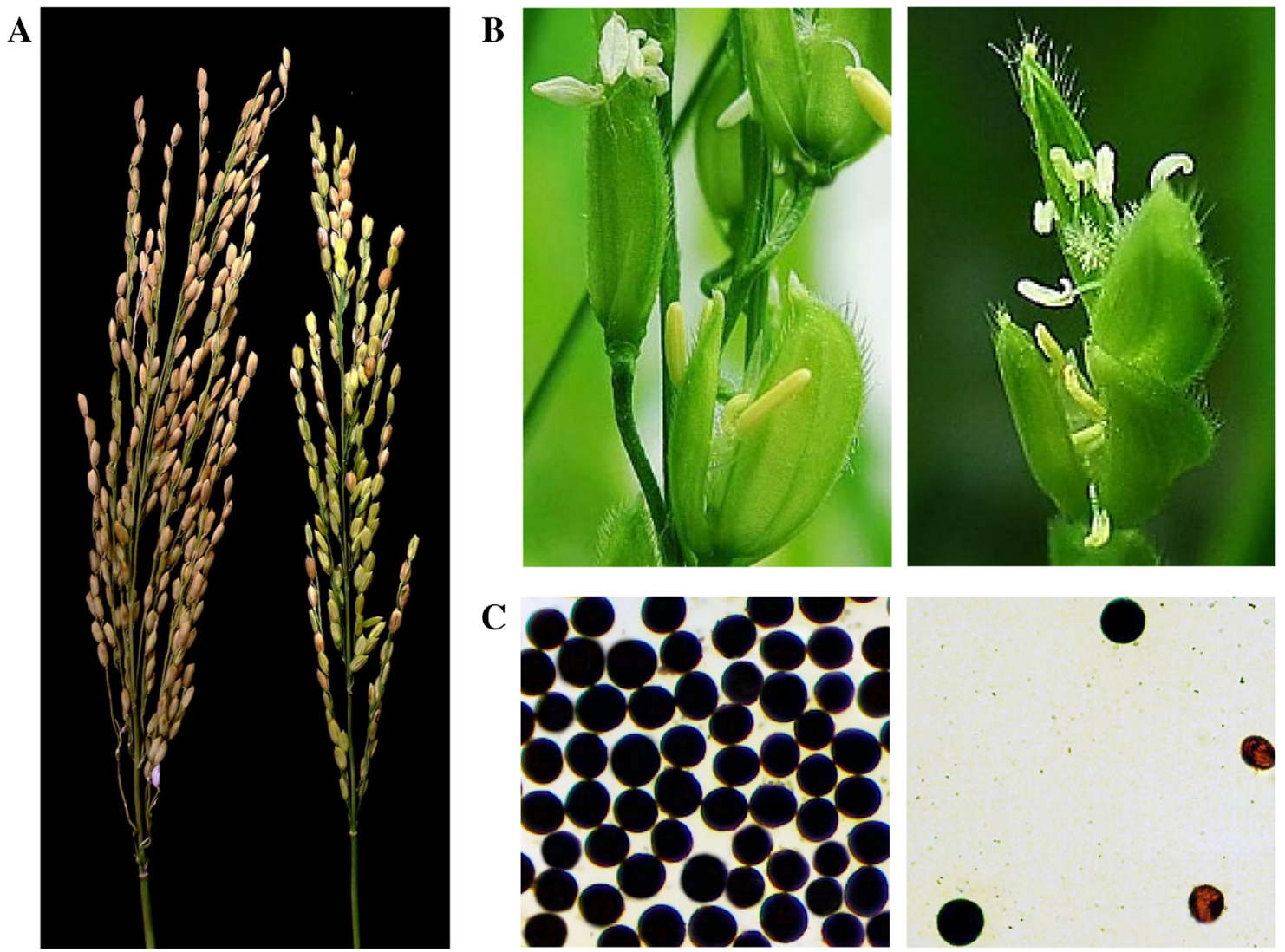

Fig. 2 Fertility performances. a Spikelet fertility of Habataki (left) and Sasanishiki (right) under E3 condition. b Flowering spikelet of Sasanishiki. Normal spikelets (left), white and light yellow curly

\section{Phenotypic performance}

The lasting high temperature at booting stage (in mid to late July, 2016) caused great damage to the mid-season rice, including many of the japonica varieties and a few of the indica varieties in Jiangxi province of China (data not shown). Sasanishiki extremely suffered from the high temperature, while Habataki was slightly affected (Fig. 2a). In E3, the mean spikelet fertility in panicles of Sasanishiki and Habataki was 19.71 and $84.36 \%$, respectively. The value of CSSLs ranged from 12.25 to $58.6 \%$ with the mean of $30.51 \%$. Similar variations were observed in E4 (Table 1). The CSSL population segregation for spikelet fertility distributed continuously in the two environments (Fig. 3). Furthermore, a significant positive correlation of spikelet fertility was found between the two environments $(r=0.462, P<0.05)$.

To understand how the high temperature at the booting stage affected spikelet fertility, we observed the flowering spikelets of Sasanishiki in the paddy field and found defects of stamen development. Compared with the normal stamens, the anthers appeared small and light yellow, or white and curly, after the high temperature stress (Fig. 2b). anthers after high temperature stress (right). c Pollen of Sasanishiki. Pollens from normal anthers $(l e f t)$, fewer pollens from high temperature treated anthers (right)

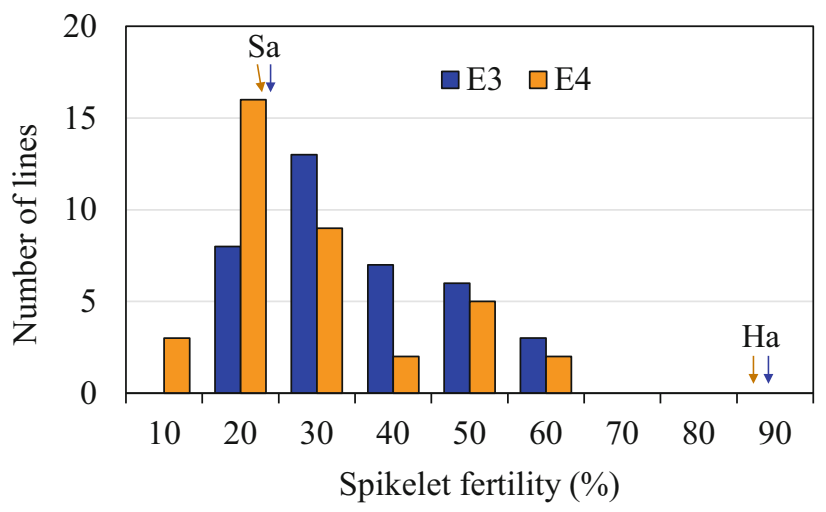

Fig. 3 Frequency distribution for spikelet fertility in the CSSLs population in E3 and E4

Moreover, the pollen stainability test revealed that the high temperature treated anthers produced much fewer pollen grains, some of which were aborted (Fig. 2c). These results indicate that the high temperature at the booting stage hindered the production of pollens and caused partial male 
sterility of Sasanishiki, which eventually led to decreased spikelet fertility.

\section{QTL mapping}

Analysis of variance (one-way ANOVA) was carried out on the spikelet fertility. Compared to Sasanishiki, a total of 14 CSSLs showed significant differences, of which, 9 CSSLs were found in one environment, the rest were identified under two environments. The additive effects ranged from -6.28 to $18.48 \%$. Most of the CSSLs exhibited significant increases in spikelet fertility, except 2 CSSLs (SL409 and SL433) (Table 2). In total, 12 QTLs were detected and mapped on 7 out of the 12 rice chromosomes (Fig. 4). Among these loci, 5 QTLs (qHTB3-1, $q H T B 3-3, q H T B 4-2, q H T B 5-1$, and $q H T B 11)$ were detected in two environments, while 7 QTLs were detected in one environment. SL419 and SL420 contained an overlapping segment on chromosome 6, so the QTL (qHTB6) was limited to the region between SSR markers RM5963 and RM3330, which was detected in E3. Two QTLs (qHTB3-2 and $q H T B 10-2$ ), with negative additive effect on HT, were detected in E4. The spikelet fertility of SL408, which harbored $q H T B 3-1$ with the largest additive effect in E3, was 58.60 and $42.07 \%$ in two environments, respectively
(Table 2). This presented a relatively large phenotypic variation between two environments. The other 4 QTLs (qHTB3-3, qHTB4-2, qHTB5-1, and qHTB11) detected in two environments, showed fairly similar effects on increasing HT.

\section{Validation of a major QTL for HT}

In order to verify the existence of the major QTL ( $q H T B 3-$ 3) for HT, three CSSLs (SL410, SL411, and SL422) were used. After high-temperature stress at the booting stage, a significant difference was observed in the spikelet fertility between two adjacent CSSLs (SL410 and SL411). SL411 performed much better than SL410 in the paddy field (Fig. 5a). SL411 and SL422 carried an overlapping segment of Habataki located between RM3525 and RM6970 on chromosome 3 (Ando et al. 2008) (Fig. 5b), and the two CSSLs showed much higher spikelet fertility than Sasanishiki. Therefore, it was believed that they contained the same QTL ( $q H T B 3-3)$, with the positive allele contributed by Habataki. To further confirm the existence of $q H T B 3-3$, BILs were developed from a cross between SL411 and Sasanishiki. Four overlapping substituted homozygous $\mathrm{F}_{3}$ lines were identified and used for phenotyping. The results showed that the spikelet fertility of the three lines (SL411-
Table 2 QTLs for spikelet fertility under high temperature detected in Sasanishiki/ Habataki CSSLs population across two environments

\begin{tabular}{|c|c|c|c|c|c|c|}
\hline QTL & Chr. & Flanking maker interval & $\mathrm{SF}(\%)$ & Add $(\%)$ & Environment & CSSL \\
\hline qHTB1 & 1 & RM1387-RM8137 & 45.30 & 12.80 & E3 & SL404 \\
\hline \multirow[t]{2}{*}{$q H T B 3-1$} & 3 & RM4108 & 58.60 & 19.45 & E3 & SL408 \\
\hline & & & 42.07 & 11.75 & E4 & \\
\hline$q H T B 3-2$ & 3 & RM5748-RM5864 & 6.87 & -5.84 & E4 & SL409 \\
\hline \multirow[t]{4}{*}{$q H T B 3-3$} & 3 & RM3525-RM6970 & 54.14 & 17.22 & E3 & SL411 \\
\hline & & & 52.73 & 17.08 & E4 & SL422 \\
\hline & & & 47.13 & 13.71 & E3 & \\
\hline & & & 44.74 & 13.09 & E4 & \\
\hline$q H T B 4-1$ & 4 & RM7585-RM5633 & 56.67 & 18.48 & E3 & SL412 \\
\hline \multirow[t]{2}{*}{$q H T B 4-2$} & 4 & RM3534-RM2431 & 48.36 & 14.33 & E3 & SL414 \\
\hline & & & 45.06 & 13.25 & E4 & \\
\hline \multirow[t]{2}{*}{$q H T B 5-1$} & 5 & RM1248-RM5579 & 48.14 & 14.22 & E3 & SL415 \\
\hline & & & 43.02 & 12.23 & E4 & \\
\hline qHTB5-2 & 5 & RM3236 & 39.67 & 9.98 & E3 & SL418 \\
\hline \multirow[t]{2}{*}{ qHTB6 } & 6 & RM5963-RM3330 & 38.44 & 9.37 & E3 & SL419 \\
\hline & & & 34.77 & 8.87 & E3 & SL420 \\
\hline qHTB10-1 & 10 & RM3882-RM4455 & 42.48 & 11.96 & E4 & SL431 \\
\hline qHTB10-2 & 10 & RM3773-RM6673 & 5.99 & -6.28 & E4 & SL433 \\
\hline \multirow[t]{2}{*}{ qHTB11 } & 11 & RM286-RM7283 & 30.86 & 6.31 & E3 & SL434 \\
\hline & & & 33.41 & 7.42 & E4 & \\
\hline
\end{tabular}

Positive additive effect means Habataki allele increasing the trait values, negative additive effect means Habataki allele decreasing the trait values

$S F$ spikelet fertility, $A d d$ additive effect 
Chr. 1

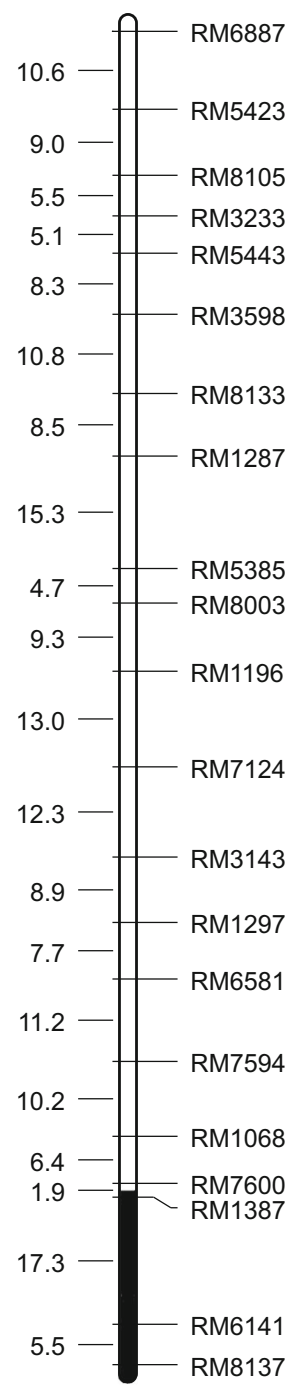

Chr. 3

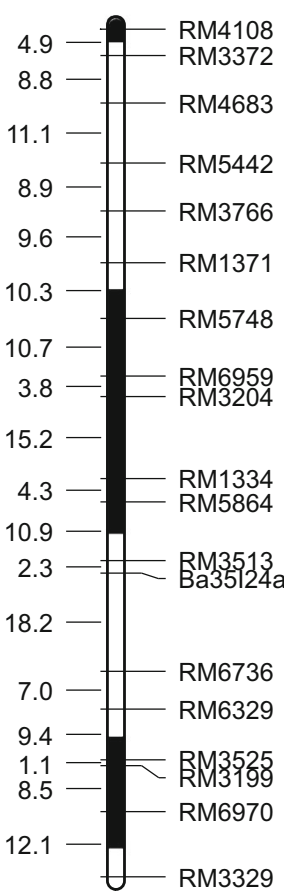

Chr. 6

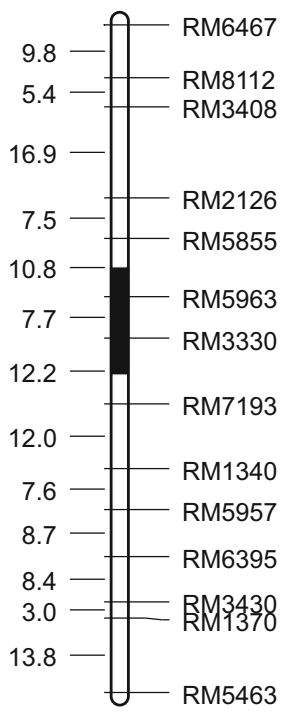

Chr. 10

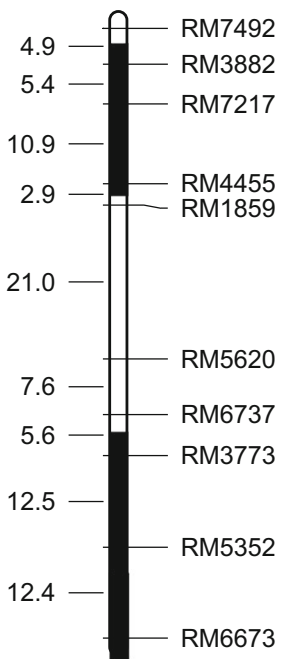

Chr. 5
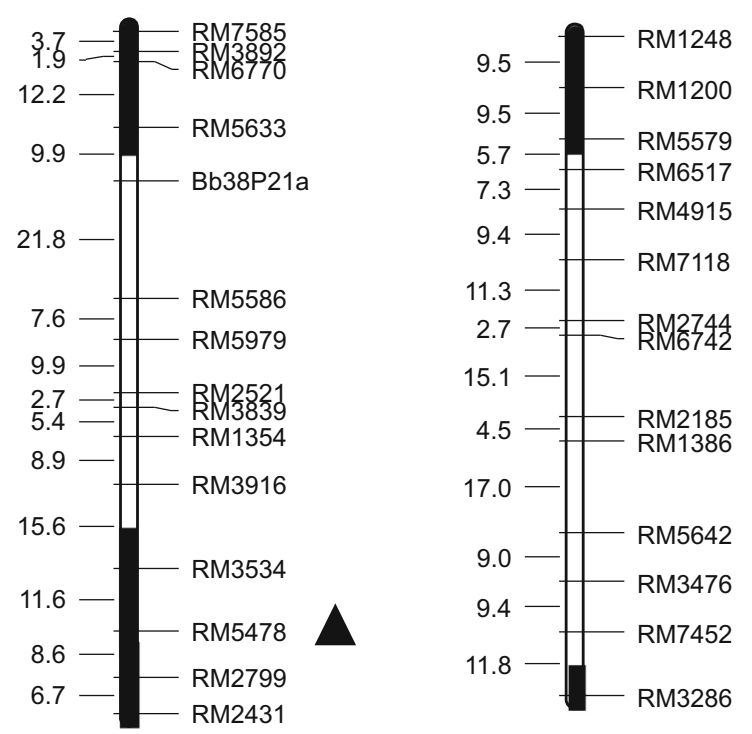

Chr. 11

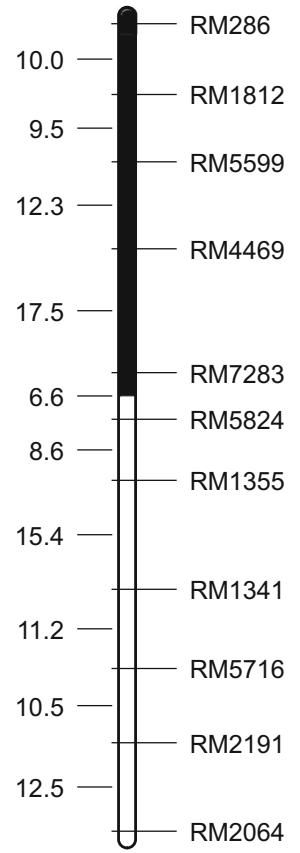

Fig. 4 QTLs for spikelet fertility under high-temperature at the booting stage. The black regions indicate the target QTL locations. The marker distance $(\mathrm{cM})$ is presented on the left side of each

L3, SL411-L5, and SL411-L16) exhibited more than twice as high as SL411-L9, which was similar to Sasanishiki, after high-temperature stress. According to genotype and phenotype data, qHTB3-3 was mapped between RM3525 and an indel marker 3-M95, approximately $2.8 \mathrm{Mb}$ apart (Fig. 5C). chromosome, and the marker names are shown on the right side. The triangles indicate that QTLs were detected in two environments

\section{Discussion}

\section{The effects of high temperature stress at the booting stage}

High temperature is already a major environmental stress limiting rice productivity. Extreme high temperatures occurred in Nanchang, Jiangxi province, from 2011 to 2016 


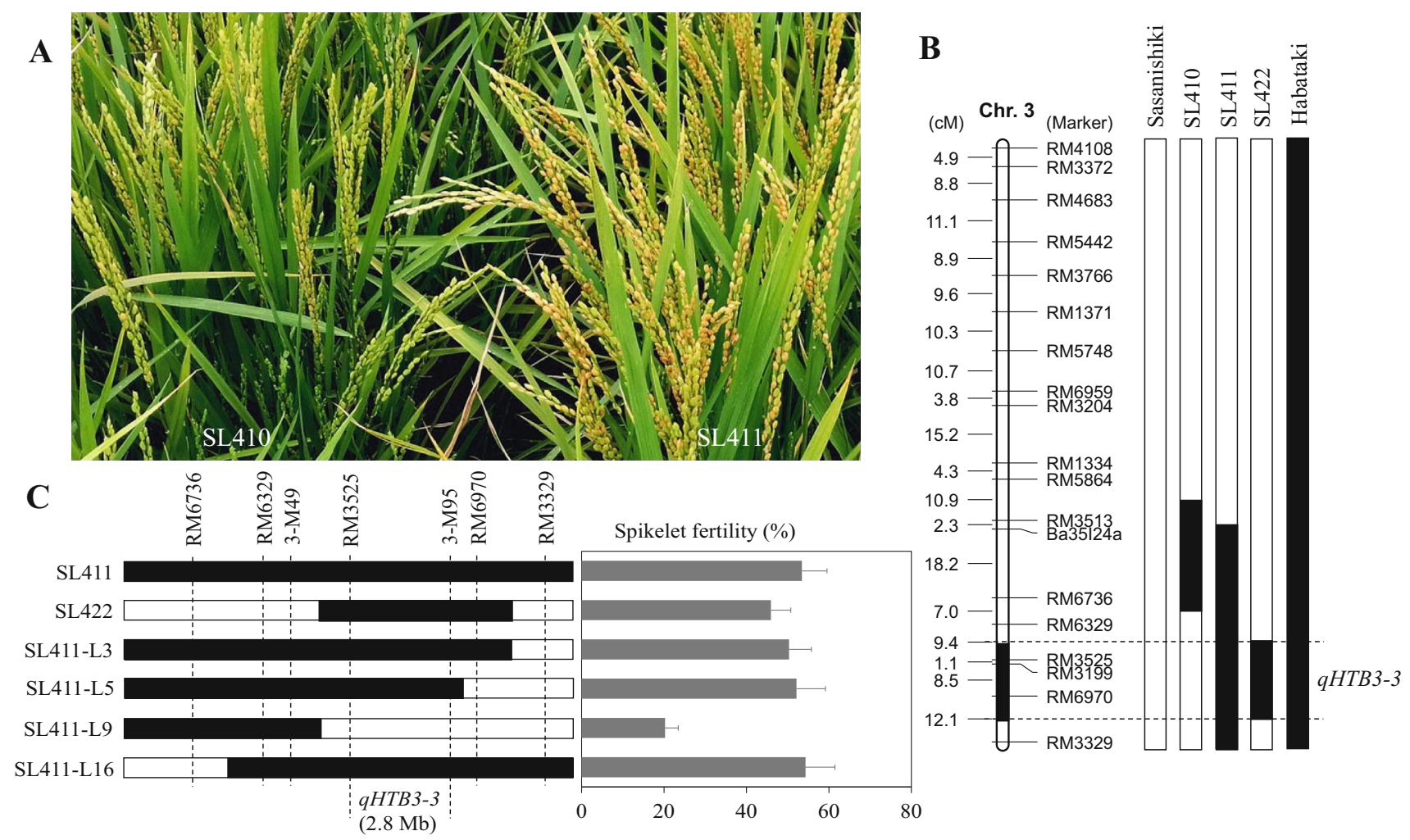

Fig. 5 Substitution mapping of $q H T B 3-3$ for high-temperature tolerance at the booting stage. a SL411 (right) showed much higher spikelet fertility than SL410 (left) in the paddy field after hightemperature stress. b Substitution mapping of qHTB3-3 on chromosome 3. The white and black regions represent Sasanishiki and

(except 2014 and 2015) according to our temperature records. The CSSL population derived from Sasanishiki and Habataki was used to test HT under natural field conditions. Bian et al. 2013, have used CSSL lines to identify QTLs for another trait (1000-grain weight PGWC). Habataki, the elite indica variety, performed well at HT in different environments at both booting and flowering stages, whereas the japonica Sasanishiki showed the opposite. The remarkable change of Sasanishiki was the anther development defects induced by high temperature stress at the booting stage. The anthers became smaller, and the number and fertility of pollen grains were decreased significantly (Fig. 2b). It was different from the performance after high temperature at the flowering stage, which showed poor anther dehiscence resulting in few germinated pollen grains on the stigma (Jagadish et al. 2010; Matsui and Omasa 2002; Prasad et al. 2006).

The characteristics caused by high temperature stress at the booting stage was very similar to the effects induced by cool-temperature damage (Oda et al. 2010; Sakata et al. 2014). The most sensitive stage to environmental stress during booting is just after meiosis (Satake and Hayase
Habataki alleles, respectively. c Graphical genotypes of 4 homozygous $\mathrm{F}_{3}$ lines derived from SL411 $\times$ Sasanishiki. $q H T B 3-3$ is indicated at the bottom (left). Mean spikelet fertility for each of the lines. Error bars represent the standard deviation (right)

1970), which is the microsporogenesis stage. The microspore and pollen grain form through meiosis and mitosis during 10-12 days before anthesis in rice. Short period of exposure to high-temperature stress at this stage could reduce spikelet fertility in a heat-sensitive rice variety (Satake and Yoshida 1978). Recently, Martínez-Eixarch and Ellis (2015) conducted an experiment under extreme high and low temperatures during reproductive development using two japonica cultivars. They found that the spikelet fertility and seed yield were severely reduced by extreme temperatures 14-7 days before anthesis. In the present study, the heading dates of Sasanishiki under two conditions were 28 and 31 July 2016, respectively. There were 13 hot days with the maximum temperature $\geq 35{ }^{\circ} \mathrm{C}$ from 21 July to 2 August 2016 that coincided with microsporogenesis.

\section{Identifying QTLs for HT at the booting stage}

To create strong subspecies heterosis, intercrossing japonica and indica has been utilized in current rice breeding practices. However, japonica cultivars usually show worse HT 
than indica cultivars. The risk of high temperature induced heat damage is possibly increased in introgression lines or new cultivars carrying japonica alleles. Therefore, it is necessary to uncover the genetic basis of HT and discover related genes, which can be applied in HT breeding to reduce the negative effects of heat. Although extreme high temperatures appear frequently, experiments on heat stress under field conditions were rarely reported. Thirty-seven CSSL lines (SL401-SL437), treated under natural high temperature, showed similar heading dates, except SL428. Additionally, there was significant segregation of spikelet fertility in the CSSL population, indicating that the field conditions used in this study were appropriate for detecting the QTLs. A total of 12 QTLs, associated with heat-tolerance at the booting stage, were identified (Table 2). They were all different from the results of Zhao et al. (2006). Compared with the QTLs detected at flowering stage in our previous study (Zhao et al. 2016), 6 QTLs (qHTB3-2, qHTB4-1, qHTB4-2, qHTB5-1, qHTB10-1, and qHTB10-2) were overlapped. Only 2 QTLs (qHTB4-2, qHTB5-1) were detected at both the booting stage and flowering stage in all environments, showing their potential breeding value. qHTB3-3 was verified to be an important QTL responsible for HT at the booting stage, however, it was not detected at the flowering stage in previous work (Zhao et al. 2016). According to the linkage map of the CSSLs, both of SL411 and SL422 carried one segment of chromosome 3 from Habataki, containing the qHTB3-3 allele (Fig. 5b). There was a difference in spikelet fertility between the two lines such that SL422 showed a little lower spikelet fertility than SL411 (Fig. 5c). It is possible that a segment of Habataki on chromosome 7 carrying in SL422 has a small negative effect on HT. Further mapping of $q H T B 3-3$ is under progress.

Acknowledgements This work was supported by the Key Program for Science and Technology of Jiangxi Province, China (20152ACF60015), and the Collaborative Innovation Program for Modern Agricultural Research of Jiangxi Province, China (JXXTCX2015001-005).

\section{References}

Ando T, Yamamoto T, Shimizu T, Ma XF, Shomura A, Takeuchi Y, Lin SY, Yano M (2008) Genetic dissection and pyramiding of quantitative traits for panicle architecture by using chromosomal segment substitution lines in rice. Theor Appl Genet 116:881-890

Bian JM, Shi H, Li CJ, Zhu CL, Yu QY, Peng XS, Fu JR, He XP, Chen XR, Hu LF, Ouyang LJ, He HH (2013) QTL mapping and correlation analysis for 1000-grain weight and percentage of grains with chalkiness in rice. J Genet 92:281-287

Buu BC, Ha PTT, Tam BP, Nhien TT, Hieu NV, Phuoc NT, Minh L, Giang LH, Lang NT (2014) Quantitative trait loci associated with heat tolerance in rice (Oryza sativa L.). Plant Breed Biotechnol 2:14-24

Chen Q, Yu S, Li C, Mou T (2008) Identification of QTLs for heat tolerance at flowering stage in rice. Sci Agric Sin 41:315-321

Eshed Y, Zamir D (1995) An introgression line population of Lycopersicon pennellii in the cultivated tomato enables the identification and fine mapping of yield-associated QTL. Genetics 141:1147-1162

Gong S, Shao F (2013) Investigation report on heat stress events for single-season rice in He county. Anhui Agric Sci Bull 19:37-38

Hirabayashi H, Sasaki K, Kambe T, Gannaban RB, Miras MA, Mendioro MS, Simon EV, Lumanglas PD, Fujita D, TakemotoKuno Y, Takeuchi Y, Kaji R, Kondo M, Kobayashi N, Ogawa T, Ando I, Jagadish KS, Ishimaru T (2015) $q E M F 3$, a novel QTL for the early-morning flowering trait from wild rice, Oryza officinalis, to mitigate heat stress damage at flowering in rice, $O$. sativa. J Exp Bot 66:1227-1236

Jagadish SVK, Craufurd PQ, Wheeler TR (2007) High temperature stress and spikelet fertility in rice (Oryza sativa L.). J Exp Bot 58:1627-1635

Jagadish SVK, Muthurajan R, Oane R, Wheeler TR, Heuer S, Bennett J, Craufurd PQ (2010) Physiological and proteomic approaches to address heat tolerance during anthesis in rice (Oryza sativa L.). J Exp Bot 61:143-156

Li XM, Chao DY, Wu Y, Huang X, Chen K, Cui LG, Su L, Ye WW, Chen H, Chen HC, Dong NQ, Guo T, Shi M, Feng Q, Zhang P, Han B, Shan JX, Gao JP, Lin HX (2015) Natural alleles of a proteasome $\alpha 2$ subunit gene contribute to thermotolerance and adaptation of African rice. Nat Genet 47:827-833

Martínez-Eixarch M, Ellis RH (2015) Temporal sensitivity of rice seed development from spikelet fertility to viable mature seed to extreme temperature stress. Crop Sci 55:354-364

Matsui T, Omasa K (2002) Rice (Oryza sativa L.) cultivars tolerant to high temperature at flowering: anther characteristics. Ann Bot 89:683-687

Oda S, Kaneko F, Yano K, Fujioka T, Masuko H, Park JI, Kikuchi S, Hamada K, Endo M, Nagano K, Nagamura Y, KawagishiKobayashi M, Suwabe K, Suzuki G, Watanabe M (2010) Morphological and gene expression analysis under cool temperature conditions in rice anther development. Genes Genet Syst $85: 107-120$

Prasad PVV, Boote KJ, Allen LH, Sheehy JE, Thomas JMG (2006) Species, ecotype and cultivar differences in spikelet fertility and harvest index of rice in response to high temperature stress. Field Crop Res 95:398-411

Rang ZW, Jagadish SVK, Zhou QM, Craufurd PQ, Heuer S (2011) Effect of high temperature and water stress on pollen germination and spikelet fertility in rice. Environ Exp Bot 70:58-65

Sakata T, Oda S, Tsunaga Y, Shomura H, Kawagishi-Kobayashi M, Aya K, Saeki K, Endo T, Nagano K, Kojima M, Sakakibara H, Watanabe M, Matsuoka M, Higashitani A (2014) Reduction of gibberellin by low temperature disrupts pollen development in rice. Plant Physiol 164:2011-2019

Satake T, Hayase H (1970) Male sterility caused by cooling treatment at the young microspore state in rice plants. 5. Estimations of pollen developmental stage and the most sensitive stage to coolness. Proc Crop Sci Soc Jpn 39:468-473

Satake T, Yoshida S (1978) High temperature-induced sterility in indica rices at flowering. Jpn J Crop Sci 47:6-17

Shah F, Huang J, Cui K, Nie L, Shah T, Chen C, Wang K (2011) Impact of high-temperature stress on rice plant and its traits related to tolerance. J Agric Sci 149:545-556

Shi CL, Jin ZQ, Zheng JC, Tang RS (2008) Effect of high temperature at meiosis stage on seed-setting rate in rice. Acta Agron Sin 34:627-631 
Shi P, Tang L, Wang L, Sun T, Liu L, Cao W, Zhu Y (2015) Postheading heat stress in rice of south China during 1981-2010. PLoS ONE 10:e0130642

Tao L, Tan H, Wang X, Cao LY, Cheng S (2007) Effects of high temperature stress on super hybird rice Guodao 6 during flowing and filling phases. Chin J Rice Sci 21:518-524

Untergasser A, Cutcutache I, Koressaar T, Ye J, Faircloth BC, Remm M, Rozen SG (2012) Primer3-new capabilities and interfaces. Nucl Acids Res 40(15):e115

Vishnu Prasanth V, Basava KR, Suchandranath Babu M, Venkata Tripura VGN, Rama Devi SJS, Mangrauthia SK, Voleti SR, Sarla N (2016) Field level evaluation of rice introgression lines for heat tolerance and validation of markers linked to spikelet fertility. Physiol Mol Biol Plants 22:179-192

Xiao YH, Pan Y, Luo LH, Zhang GL, Deng HB, Dai LY, Liu XL, Tang WB, Chen LY, Wang GL (2011) Quantitative trait loci associated with seed set under high temperature stress at the flowering stage in rice (Oryza sativa L.). Euphytica 178:331-338

Yang BY, Shen SH, Tao SL, Qian L, Zou XZ (2012) Spatial and temporal pattern of rice heat injury in Jiangxi. Chin J Agrometeorol 33:615-622
Ye C, Argayoso MA, Redoña ED, Sierra SN, Laza MA, Dilla CJ, Mo Y, Thomson MJ, Chin J, Delaviña CB, Diaz GQ, Hernandez JE (2012) Mapping QTL for heat tolerance at flowering stage in rice using SNP markers. Plant Breed 131:33-41

Ye C, Tenorio FA, Argayoso MA, Laza MA, Koh HJ, Redoña ED, Jagadish KS, Gregorio GB (2015) Identifying and confirming quantitative trait loci associated with heat tolerance at flowering stage in different rice populations. BMC Genet 16:41

Yin X, Kropff MJ, Goudriaan J (1996) Differential effects of day and night temperature on development to flowering in rice. Ann Bot 77:203-213

Zhang T, Yang L, Jiang KF, Huang M, Sun Q, Chen WF, Zheng JK (2008) QTL mapping for heat tolerance of the tassel period of rice. Mol Plant Breed 6:867-873

Zhao Z, Jiang L, Xiao Y, Zhang W, Zhai H, Wan J (2006) Identification of QTLs for heat tolerance at the booting stage in rice (Oryza sativa L.). Acta Agron Sin 32:640-644

Zhao L, Lei J, Huang Y, Zhu S, Chen H, Huang R, Peng Z, Tu Q, Shen X, Yan S (2016) Mapping quantitative trait loci for heat tolerance at anthesis in rice using chromosomal segment substitution lines. Breed Sci 66:358-366 